\title{
BMJ Open Acute effects of breaking up prolonged sitting on fatigue and cognition: a pilot study
}

Patrik Wennberg, ${ }^{1}$ Carl-Johan Boraxbekk, ${ }^{2}$ Michael Wheeler, ${ }^{3,4}$ Bethany Howard, ${ }^{3,5}$ Paddy C Dempsey, ${ }^{3,5}$ Gavin Lambert, ${ }^{3,5}$ Nina Eikelis, ${ }^{3}$ Robyn Larsen, ${ }^{3}$ Parneet Sethi, ${ }^{3}$ Jessica Occleston, ${ }^{3}$ Jenny Hernestål-Boman, ${ }^{1}$ Kathryn A Ellis, ${ }^{6}$ Neville Owen, ${ }^{3,5,7,8}$ David W Dunstan ${ }^{3,4,5,7,8,9,10,11}$

To cite: Wennberg $P$, Boraxbekk C-J, Wheeler M, et al. Acute effects of breaking up prolonged sitting on fatigue and cognition: a pilot study. BMJ Open 2016:6:e009630.

doi:10.1136/bmjopen-2015009630

- Prepublication history and additional material is available. To view please visit the journal (http://dx.doi.org/ 10.1136/bmjopen-2015009630).

Received 4 August 2015 Revised 8 December 2015 Accepted 22 December 2015

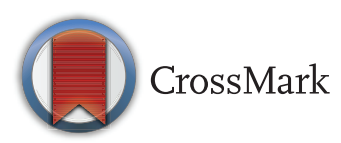

For numbered affiliations see end of article.

Correspondence to Dr Patrik Wennberg; patrik.wennberg@umu.se

\section{ABSTRACT}

Objectives: To compare the acute effects of uninterrupted sitting with sitting interrupted by brief bouts of light-intensity walking on self-reported fatigue, cognition, neuroendocrine biomarkers and cardiometabolic risk markers in overweight/obese adults.

Design: Randomised two-condition crossover trial. Setting: Laboratory study conducted in Melbourne, Australia.

Participants: 19 overweight/obese adults (45-75 years).

Interventions: After an initial $2 \mathrm{~h}$ period seated, participants consumed a meal-replacement beverage and completed (on 2 days separated by a 6-day washout period) each condition over the next $5 \mathrm{~h}$ : uninterrupted sitting (sedentary condition) or sitting with 3 min bouts of light-intensity walking every $30 \mathrm{~min}$ (active condition).

Primary outcome measures: Self-reported fatigue, executive function and episodic memory at $0 \mathrm{~h}, 4 \mathrm{~h}$ and $7 \mathrm{~h}$.

Secondary outcome measures: Neuroendocrine biomarkers and cardiometabolic risk markers (blood collections at $0 \mathrm{~h}, 4 \mathrm{~h}$ and $7 \mathrm{~h}$, blood pressure and heart rate measured hourly and interstitial glucose measured using a continuous glucose monitoring system).

Results: During the active condition, fatigue levels were lower at $4 \mathrm{~h}(-13.32(95 \% \mathrm{Cl}-23.48$ to -3.16$))$ and at $7 \mathrm{~h}(-10.73(95 \% \mathrm{Cl}-20.89$ to -0.58$))$ compared to the sedentary condition. Heart rate was higher at $4 \mathrm{~h}$ $(4.47(95 \% \mathrm{Cl} 8.37$ to 0.58$))$ and at $7 \mathrm{~h}(4.32(95 \% \mathrm{Cl}$ 8.21 to 0.42$)$ ) during the active condition compared to the sedentary condition. There were no significant differences between conditions by time for other variables. In the sedentary condition, changes in fatigue scores over time correlated with a decrease in heart rate and plasma dihydroxyphenylalanine (DOPA) and an increase in plasma dihydroxyphenylglycol (DHPG).

Conclusions: Interrupting prolonged sitting with lightintensity walking breaks may be an effective fatigue countermeasure acutely. Fatigue levels corresponded with the heart rate and neuroendocrine biomarker changes in uninterrupted sitting in this pilot study. Further research is needed to identify potential implications, particularly for the occupational health context.

\section{Strengths and limitations of this study}

- This is the first experimental study to compare the acute effects of uninterrupted sitting on subjective fatigue and cognitive functioning with sitting interrupted every $30 \mathrm{~min}$ with lightintensity walking in a $7 \mathrm{~h}$ 'whole working day' model.

- A randomised crossover study design was used.

- Given that this is a pilot study, the sample size may have limited the ability to detect an effect from breaking up sitting in several of the outcome measures.

- The longer term relevance of acute effects of breaking up prolonged sitting is uncertain.

Trial registration number: ACTRN12613000137796; Results.

\section{INTRODUCTION}

There is accumulating evidence on the negative health consequences of prolonged sitting. Previous experimental studies have demonstrated that uninterrupted sitting is deleteriously associated with metabolic parameters, ${ }^{1}{ }^{2}$ blood pressure ${ }^{3}$ and markers of haemostasis ${ }^{4}$ when compared to sitting that is interrupted with short bouts of physical activity. A recent experimental trial utilising a $7 \mathrm{~h}$ 'whole working day' model found that in overweight and obese adults, regularly interrupting sitting time with short bouts of either light-intensity or moderate-intensity walking lowered postprandial glucose and insulin concentrations when compared with prolonged sitting. ${ }^{1}$ Although glucose ingestion has been suggested to acutely improve memory and cognitive function, ${ }^{5}$ meals that trigger postprandial hyperglycaemia have been associated with a decline in cognitive function relative to meals that cause a more 
gradual rise in blood glucose concentrations. ${ }^{6}$ The emerging evidence relating changes in postprandial glucose to changes in cognitive function provides a rationale to investigate whether breaking up prolonged sitting with light-intensity physical activity may also be relevant for cognitive performance.

Aside from the metabolic benefits, physical activity may mediate several putative pathways involved in general mental fatigue and cognition. These include increased cerebral blood flow ${ }^{7}$ and expression of neurotrophins such as brain-derived neurotrophic factor (BDNF), ${ }^{8}$ which have been linked to both acute neuronal activation and long-term functional and structural changes in the brain. ${ }^{9}$ During physical activity, particularly of higher intensities, there is a release of epinephrine and norepinephrine from the adrenal medulla. Also, peripheral catecholamine release has a direct effect on synthesis and release of norepinephrine in the brain via activation of adrenoreceptors on vagal afferent nerves ${ }^{10}$ that may increase alertness and facilitate cognition. Some sedentary behaviours, such as TV viewing, have been linked to adverse long-term effects such as detrimental cognitive development in early childhood ${ }^{11}$ and poorer cognitive function in older adults, ${ }^{12}$ but the mediating pathways are not well understood.

The possible influence of prolonged sitting on cognition is of great potential importance for work productivity and cognitive health, especially among office workers who are particularly vulnerable to prolonged uninterrupted sedentary behaviour. ${ }^{13}$ In previous research, office workers have reported reduced fatigue across the day with the use of sit-to-stand desks when compared to seated work, ${ }^{14}{ }^{15}$ but also self-perceived hampered concentration and focus when sitting and standing work were combined. ${ }^{14}$ Given this preliminary evidence, and from a physiological standpoint, we hypothesise that the pattern of intermittent short bouts of physical activity leading to reduced prolonged sedentary states may confer greater benefit on fatigue and cognitive performance than uninterrupted sitting. We examined, in a $7 \mathrm{~h}$ 'whole working day' model, the acute effects of uninterrupted sitting on subjective fatigue and cognitive functioning (executive functions and episodic memory), compared with sitting interrupted every $30 \mathrm{~min}$ with light-intensity walking. The study had an explorative approach and a small sample size and was conducted as an evaluation of technical procedures and logistic feasibility of a full-scale study (clinical trial registration ACTRN12614000737639).

\section{METHODS}

\section{Study design}

The study was a laboratory-based randomised crossover trial with two experimental conditions separated by a 6-day washout period. All participants provided signed informed consent.

\section{Participants}

Participants were recruited from the general community between May and August 2013 through the use of study fliers and posters or were contacted on the basis of their previously registered expression of interest in future studies (see online supplementary figure). Eligible participants were aged $45-75$ years with a body mass index (BMI) $\geq 25 \mathrm{~kg} / \mathrm{m}^{2}$ but $<40 \mathrm{~kg} / \mathrm{m}^{2}$, who reported sitting $>5 \mathrm{~h}$ a day and were not engaged in regular moderate-to-vigorous intensity physical activity (MVPA; $\geq 150 \mathrm{~min} /$ week for $>3$ months). Exclusion criteria included those who were: non-English speaking, pregnant, currently smoking, diagnosed with diabetes, reported to have cognitive or physical problems that may limit the ability to perform the activity bouts or complete the computer-based cognitive test programme, currently using any of the following medications: glucose-lowering, lipid-lowering, antidepressant or oral cortisone medication. Consistent with a previous trial with a similar experimental design, ${ }^{1} 19$ participants were recruited.

\section{Randomisation}

Participants were randomised to one of two possible trial-condition orders using fixed block sizes for men and women. The computer-generated randomisation list and randomisation envelopes were prepared and kept by a third party. Research staff opened randomisation envelopes once informed consent was obtained.

\section{Pre-experimental procedure}

\section{Screening and familiarisation}

Following telephone screening, participants attended an assessment and familiarisation visit at the research laboratory. During this visit, they underwent training on the cognitive test battery and were given the opportunity to ask questions and were allowed unlimited time to complete practice tests to ensure sufficient understanding of each task. They were then familiarised with walking on the motorised treadmill on a level incline.

\section{Diet and physical activity}

To control and account for any diet-induced variability in study outcomes, participants were provided with standardised meal packs for consumption on the evening prior to each experimental condition. The meal packs (consisting of a drink, snack and a commercially available microwave meal) were individualised to meet $33.3 \%$ of daily estimated energy requirements using the Schofield equation ${ }^{16}$ with a 1.5 physical activity factor and a target macronutrient profile of $12-15 \%$ energy from protein, 55-58\% energy from carbohydrate and 29-31\% energy from fat. To minimise the potential carryover effects of physical activity, participants were instructed to avoid any moderate and/or vigorous physical activity for at least $48 \mathrm{~h}$ prior to each experimental condition. Sitting time and MVPA were assessed with an accelerometer, Actigraph GT3X+ (ActiGraph LLC, Pensacola, Florida, USA), which was worn on the hip 
during waking hours. The participants wore the accelerometer starting from the familiarisation visit until the end of the second experimental day. Wear time and activity type, duration and intensity undertaken during any non-wear periods were recorded in activity diaries. Accelerometer data were downloaded using ActiLife 6.5.4 software and summarised using SAS V.9.4 for each of the habitual days prior to the condition. Non-wear periods were deleted for analyses; these were periods with at least $60 \mathrm{~min}$ of zero counts per minute (cpm), allowing for up to two consecutive, $1 \mathrm{~min}$ interruptions (count values between 1-49 cpm) per non-wear period. ${ }^{17}$ Activity counts were categorised as sedentary (<100 cpm; predominantly sitting), ${ }^{18}$ light-intensity activity (100-1951 cpm; typically gentle walking) ${ }^{19}$ or MVPA ( $\geq 1952 \mathrm{cpm}$; typically at least brisk walking). ${ }^{19}$ Average of sitting time and MVPA were calculated for each individual across valid days (days where the monitor was worn for at least $600 \mathrm{~min}$ ).

\section{Experimental procedure}

The study protocol is shown in figure 1. After having fasted overnight, participants arrived at the laboratory between 7:00 and 8:00 hours. In both conditions, participants remained seated for the initial $2 \mathrm{~h}$ to ensure that a sedentary status was achieved prior to the consumption of a mixed meal. The mixed meal was provided as a $200 \mathrm{~mL}$ drink containing $50 \mathrm{~g}$ fat (Calogen; Nutricia, Australia) and $75 \mathrm{~g}$ carbohydrate (100\% maltodextrin powder; Natural Health, Australia). This meal was chosen to be consistent with previous work that revealed differences in postprandial glucose and insulin levels with prolonged sitting when compared with to an equivalent duration of sitting that is interrupted with short bouts of light-intensity walking. ${ }^{1}$ A small snack (Oatmeal biscuits; Paradise, Australia) was also provided to participants at $5 \mathrm{~h}$ to circumvent any unwanted effects from postprandial hypoglycaemia.
After the mixed meal, participants followed their respective trial condition protocols for the following $5 \mathrm{~h}$ under the direct supervision of research staff. In the sedentary condition, participants remained seated during the experimental period, only rising from the chair to visit the toilet (on the same floor, approximately $15 \mathrm{~m}$ from the chair). During the sedentary condition, 14 participants visited the toilet (8 participants on one occasion, 3 participants on two occasions, 2 participants on three occasions and 1 participant on four occasions) compared to the active condition where 8 participants visited the toilet only once.

In the active condition, participants rose from the seated position every $30 \mathrm{~min}$ throughout the experimental period and completed a 3 min bout of light-intensity walking on a motorised treadmill (Nautilus T916) at a level incline $(0$ gradient $)$ at $3.2 \mathrm{~km} / \mathrm{h}$. They then returned to the seated position. This procedure was undertaken on 10 occasions, providing a total of $30 \mathrm{~min}$ of light-intensity activity. Previous research has shown that a $2 \mathrm{~min}$ bout of light-intensity walking every $20 \mathrm{~min}$ lowered postprandial glucose and insulin concentrations, ${ }^{1}$ but the intervals were expanded in the current study to enable a complete session of fatigue and cognitive assessments during a single bout of sitting.

At $0 \mathrm{~h}, 4 \mathrm{~h}$ and $7 \mathrm{~h}$, participants completed the visual analogue scale for fatigue (VAS-F) ${ }^{20}$ directly followed by the cognitive test battery (described under 'Cognitive assessment' below) (total time: $26 \pm 1 \mathrm{~min}$ ). The cognitive tests were conducted using E-prime 2.0 (Psychology Software Tools Inc) on a Toshiba Satellite Pro C650 laptop computer with a $1366 \times 768,15.6$ inch screen in a seated position. Throughout both conditions, participants read books, magazines or newspapers, watched television or DVDs or worked on a laptop computer. However, participants were instructed to limit the amount of interactive multimedia or problem-solving tasks to avoid impact on the cognitive testing. Blood

Figure 1 Study protocol. 
collections, fatigue and cognitive tests (in that order) were undertaken at $0 \mathrm{~h}, 4 \mathrm{~h}$ and $7 \mathrm{~h}$.

\section{Fatigue assessment}

VAS for fatigue

A VAS to evaluate fatigue severity (VAS-F) was used during the experimental days. The VAS-F questionnaire consisted of 18 items related to the subjective experience of fatigue and energy and has shown high reliability (Cronbach's $\alpha$ 0.91-0.96), sensitivity for changes in fatigue over a day, and compares favourably with the Stanford Sleepiness Scale and the Profile of Mood States in healthy participants. ${ }^{20}$ Each item asks participants to mark with a single vertical dash, representing how they currently feel, on a continuous horizontal line that extends between two extremes, for example from 'not at all tired' to 'extremely tired'.

\section{Cognitive assessment}

A battery of cognitive tests covering functions including episodic memory, inhibition and updating were used. The focus on central executive tasks was chosen on the basis of previously described larger effect size for higherorder cognitive tasks compared to less complex tasks. ${ }^{21} 22$ To test episodic memory function, a face-name association test was used. ${ }^{23}$ Participants were instructed to remember a fictional name associated with an unknown face. During retrieval, they were presented with the previously viewed faces together with three letters. The task was to indicate which of the three letters corresponded to the first letter of the name that was associated with the face. To test the executive function of inhibition, two tests were used, the Eriksen flanker task ${ }^{24}$ and a modified Stroop colour-word task. ${ }^{25}$ In the Eriksen flanker task, the participants indicated the direction of the middle arrow in either congruent ( $\gg \gg \gg)$ ) or incongruent $(>><>>)$ stimuli by pressing a button as fast and as accurately as possible. In the Stroop test, participants were asked to indicate the colour a colour-word was written in, for example, 'blue' written in the colour blue, responding as fast and as accurately as possible.

The executive function updating was also tested using two tests: n-back ${ }^{26}$ and letter memory. ${ }^{27}$ In n-back, the participants were asked to indicate whether each number (1-9) in a list matched a number occurring one, two or three numbers back. Accuracy and response time were used as dependent measures. In letter memory, they viewed lists consisting of serially presented letters (A-D, $2 \mathrm{~s}$ per letter). They did not know the length of each list. When the list ended, they were asked to recall the last four presented letters in the correct order as fast and as accurately as possible. A cognitive composite score (Z-score transformed) was derived from the five cognitive subtests.

\section{Continuous glucose monitoring}

Glucose concentrations were recorded using an IPro2 (Medtronic) Continuous Glucose Monitoring System
(CGMS) ${ }^{28} 29$ during both experimental conditions. The CGMS sensor was inserted under the skin in the lower back, lateral to the medioclavicular line between the iliac crest and the lowest rib, and measured interstitial glucose concentrations every $5 \mathrm{~min}$. Capillary fingerpricks (Optium Xceed) at $0 \mathrm{~h}, 1 \mathrm{~h}$ and $4 \mathrm{~h}$ were used for calibration in accordance with the manufacturer's instructions. The total area under the glycaemic response curve (total AUC) and the net incremental AUC (net iAUC), which includes all incremental area below the curve, including the area below the fasting concentration, was derived from the CGMS measurements.

\section{Plasma analysis}

Fasting and postprandial blood samples were collected from a venous cannula inserted into the lower arm. Blood was transferred in EDTA tubes for BDNF, insulin, interleukin 6, cortisol and in Glutathione (GSH)/ Ethylene Glycol Tetraacetic Acid (EGTA) tubes for analysis of catechols after at least $10 \mathrm{~min}$ of seated rest following the cannulation. Samples were immediately put on ice, then centrifuged ( $1800 \mathrm{rpm}$ for $20 \mathrm{~min}$ at $4^{\circ} \mathrm{C}$ ) within $30 \mathrm{~min}$ of collection, and plasma was stored at $-80^{\circ} \mathrm{C}$ for later analysis. To avoid intra-assay variability, analyses of biochemical markers from the EDTA plasma were conducted in a single batch after the study. Catechols, however, were analysed within 2 months in three batches to minimise the risk of degradation from long-term storage. Plasma catechols were determined by high-performance liquid chromatography with coulometric detection, following extraction from plasma using alumina adsorption. ${ }^{30}$

\section{Blood pressure and heart rate measurements}

Blood pressure and heart rate were measured hourly using an automated oscillometric blood pressure monitor (Digital Automatic Blood Pressure Monitor HEM-907, Omron, Kyoto, Japan) as the average of three single measurements. The measurements were taken at 5 min before each walking bout in a seated upright position on the arm contralateral to the arm with the intravenous cannula.

\section{Statistical analysis}

Generalised linear mixed models (GLMMs) with a single random intercept (participant) were used to examine the differential effects of the experimental conditions in variables measured continuously (interstitial glucose), hourly and at $0 \mathrm{~h}, 4 \mathrm{~h}$ and $7 \mathrm{~h}$. All analyses were adjusted for time and order effects and interstitial glucose changes were also adjusted for capillary fasting glucose concentration and BMI. Temporal changes between conditions were also assessed by including a condition-time interaction term. Pearson's pairwise correlation tests were used to assess the relationship of fatigue to plasma markers. A probability level of 0.05 was adopted. All statistical analyses were performed using 
Table 1 Characteristics of the 19 participants in the study (10 men and 9 women)

\begin{tabular}{lc}
\hline BMI $\left(\mathrm{kg} / \mathrm{m}^{2}\right)$ & $31.5(4.7)$ \\
Waist circumference (cm) & $105.2(12.4)$ \\
Completed university degree* & 78.9 \\
Taking antihypertensive medication* & 47.4 \\
Habitual moderate-to-vigorous physical & $35.80(30.86)$ \\
activity (min/day) $\dagger$ & \\
Habitual sitting time (h/day) $\dagger$ & $9.82(2.19)$ \\
Sleep time (h/night)* & $7.4(0.8)$ \\
\hline Data are \% or mean (SD). & \\
${ }^{*}$ Assessed using self-reports. & \\
†Assessed using activity monitors during the washout period \\
between the two experimental days. \\
BMI, body mass index.
\end{tabular}

Stata V.12 for Windows (StataCorp LP). Data are reported as mean $( \pm \mathrm{SD})$ in the text and tables and as estimated marginal means \pm SEM in the figures unless otherwise indicated.

\section{RESULTS}

All 19 participants (mean age $59.7 \pm 8.1$ years; 10 men $/ 9$ women) completed both trial conditions and were included in the analyses. Participant characteristics are shown in table 1.

According to the accelerometer data, participants engaged in a modest amount of daily MVPA during the washout period. Levels of MVPA in the $48 \mathrm{~h}$ prior to each experimental condition did not differ significantly $(36.29 \pm 29.20 \mathrm{~min} /$ day before the sedentary condition vs $30.18 \pm 42.16 \mathrm{~min} /$ day before the active condition; $\mathrm{p}=0.607)$.

The mean rating of perceived exertion (RPE), assessed by the Borg RPE scale, ${ }^{31}$ for the walking bouts was 9.1 \pm 2.0 (9 corresponds with 'Very light' on the Borg RPE scale). Within-condition and between-condition responses for the fatigue score, cognitive scores and biomarkers at $0 \mathrm{~h}, 4 \mathrm{~h}$ and $7 \mathrm{~h}$ are presented in tables 2 and 3 , respectively. An effect of time was seen for fatigue (at
$4 \mathrm{~h}, \mathrm{p}=0.008$; at $7 \mathrm{~h}, \mathrm{p}=0.010)$, cognitive composite score (at $7 \mathrm{~h}, \mathrm{p}=0.033$ ) and heart rate (at $4 \mathrm{~h}, \mathrm{p}<0.001$; at $7 \mathrm{~h}$, $\mathrm{p}<0.001)$ in both conditions combined. During the active condition, fatigue levels were lower at $4 \mathrm{~h}(-13.32$ $(95 \% \mathrm{CI}-23.48$ to -3.16$))$ and at $7 \mathrm{~h}(-10.73(95 \% \mathrm{CI}$ -20.89 to -0.58$)$ ) compared to the sedentary condition (see figure 2). Heart rate was higher at $4 \mathrm{~h}(4.47(95 \%$ CI 8.37 to 0.58$))$ and at $7 \mathrm{~h}(4.32(95 \%$ CI 8.21 to 0.42$))$ during the active condition compared to the sedentary condition. A trend $(p=0.077)$ for improvement in the episodic memory test was observed in the active condition compared to the sedentary condition. No statistically significant differences were seen for any of the other measured variables, including the individual executive function tests (results are only shown for the composite scores), interstitial glucose total AUC $(\mathrm{p}=0.48)$ and net iAUC $(p=0.14)$. The changes in fatigue score over time correlated with a decrease in heart rate $\left(0-4 \mathrm{~h}: \mathrm{r}_{\mathrm{s}}=-0.60\right.$, $\mathrm{p}=0.007)$ and plasma level of DOPA $\left(0-4 \mathrm{~h}: \mathrm{r}_{\mathrm{s}}=-0.59\right.$, $\mathrm{p}=0.009)$ and an increase in plasma level of DHPG (0$\left.4 \mathrm{~h}: \mathrm{r}_{\mathrm{s}}=0.73, \mathrm{p}<0.001 ; 0-7 \mathrm{~h}: \mathrm{r}_{\mathrm{s}}=0.47, \mathrm{p}=0.040\right)$ in the sedentary condition (but not in the active condition).

\section{DISCUSSION}

Increases in fatigue levels observed during uninterrupted sitting were attenuated significantly by intermittent light-intensity walking. A non-significant trend for an improvement in cognitive performance with lightintensity walking breaks was observed. The difference in fatigue between the two conditions was observed after $4 \mathrm{~h}$ and, interestingly, persisted after $7 \mathrm{~h}$ (even though stabilisation in fatigue score was seen between $4 \mathrm{~h}$ and $7 \mathrm{~h}$ in the sedentary condition). The increase in fatigue during uninterrupted sitting correlated with a decrease in heart rate and changes in plasma levels of catechols (acute increase of DHPG and a decrease of DOPA). As a comparison, the magnitude of change in fatigue score from $0 \mathrm{~h}$ to $4 \mathrm{~h}$ during uninterrupted sitting corresponds to approximately $40 \%$ of the difference in

Table 2 Within-condition responses for the fatigue and cognitive scores at 0,4 and $7 \mathrm{~h}$

\begin{tabular}{|c|c|c|c|c|c|c|}
\hline \multirow[b]{2}{*}{ Score } & \multirow[b]{2}{*}{ Condition } & \multirow[b]{2}{*}{$\mathbf{N}$} & \multicolumn{3}{|l|}{ Time (h) } & \multirow[b]{2}{*}{ p Value* } \\
\hline & & & 0 & 4 & 7 & \\
\hline \multirow[t]{3}{*}{ Fatigue score } & Active & 19 & $37.9(18.19)$ & $34.3(17.28)$ & 36.6 (19.09) & \\
\hline & Sedentary & 19 & $37.4(16.0)$ & $47.1(18.84)$ & $46.8(18.42)$ & \\
\hline & & & & & & 0.024 \\
\hline Cognitive composite & Active & 19 & $0.018(0.52)$ & $0.080(0.45)$ & $0.273(0.28)$ & \\
\hline score & Sedentary & 19 & $-0.016(0.50)$ & $0.058(0.33)$ & $0.156(0.41)$ & 0.669 \\
\hline \multicolumn{7}{|l|}{ Cognitive subtests } \\
\hline Executive functions & Active & 19 & $-0.002(0.49)$ & $0.127(0.43)$ & $0.171(0.30)$ & \\
\hline composite score & Sedentary & 19 & $0.003(0.45)$ & $0.079(0.36)$ & $0.154(0.39)$ & 0.886 \\
\hline \multirow[t]{2}{*}{ Episodic memory score } & Active & 19 & $68.9(10.70)$ & $64.5(13.84)$ & 78.5 (11.6) & \\
\hline & Sedentary & 19 & $65.4(14.77)$ & $66.2(11.61)$ & $69.3(11.8)$ & 0.077 \\
\hline
\end{tabular}

Bold typeface indicates significance at $p<0.05$.

${ }^{*} p$ Values for difference in temporal changes by condition. 


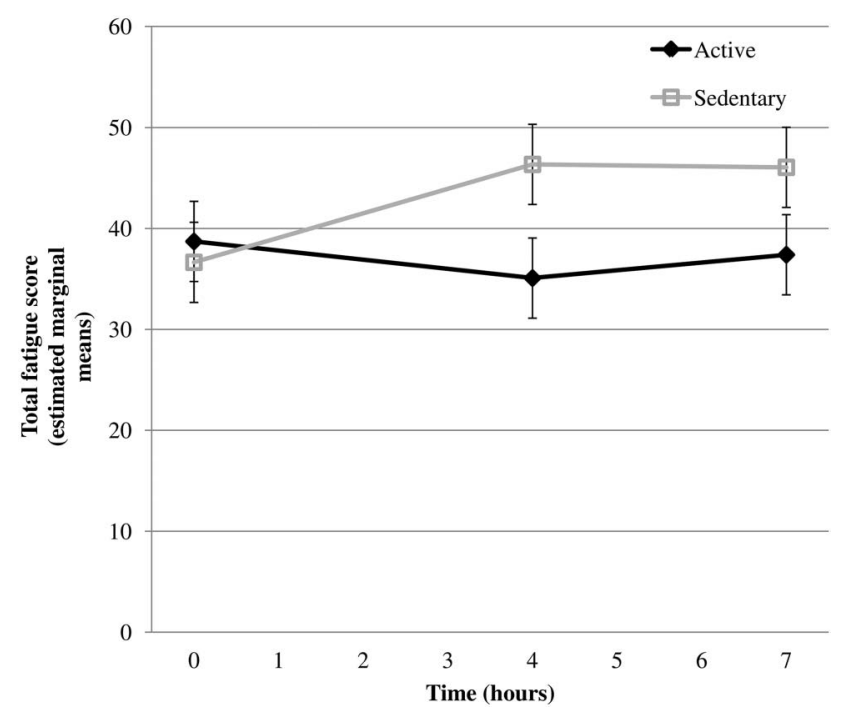

Figure 2 Total fatigue score (estimated marginal means) at 0,4 and $7 \mathrm{~h}$ for sedentary and active conditions.

fatigue score recorded before and after one night's sleep in a study of 75 healthy individuals aged $18-55$ years. ${ }^{20}$

Previous research on the relationship between sedentary behaviour and fatigue in adults is scarce. Ellingson and colleagues ${ }^{32}$ reported that women who were categorised as being insufficiently active $(<150 \mathrm{~min} /$ week $)$, but who also spent less time sedentary $(\leq 1 \mathrm{~h} /$ day, measured by accelerometer), had significantly lower levels of fatigue compared with their more sedentary peers. Our findings are consistent with those of Thorp et al, ${ }^{14}$ who observed significant attenuations in fatigue levels in office workers transitioning from a seated to a standing work posture every $30 \mathrm{~min}$ across the workday, relative to seated work. These findings add to the existing literature by providing initial experimental evidence that the relationship of prolonged uninterrupted sitting and fatigue may be causal and by examining the effect of short intermittent light-walking breaks on increased fatigue.

Fatigue is a multifaceted phenomenon. Acute fatigue can be viewed as a normal and protective mechanism to physical and/or mental exertion, which usually decreases as the exertion recedes. In contrast, persistent fatigue is associated with several medical and psychiatric illnesses as well as impaired cognitive performance ${ }^{33}$ and is one of the most common symptoms in community and primary care settings. ${ }^{34}$ The underlying neurobiological mechanisms that may be responsible for the increased fatigue from prolonged sitting and attenuated fatigue from intermittent walking breaks observed in this study are unclear. However, studies in individuals with chronic fatigue have found several alterations and/ or dysfunctions in nervous and endocrine systems, ${ }^{35}$ such as impaired autonomic nervous functions. ${ }^{36}$ Interestingly, studies have also found autonomic nervous alterations (detected via electrocardiography) associated with daily levels of fatigue in healthy individuals after a 30 min fatigue-inducing mental task session. ${ }^{37}$
The increased fatigue observed during uninterrupted sitting corresponded with a lower heart rate, decreased plasma level of DOPA (a precursor of the catecholamines dopamine, epinephrine and norepinephrine) and increased plasma level of DHPG (a deaminated metabolite of norepinephrine). The changes in heart rate and plasma levels of these compounds may reflect alterations in the sympathetic nervous system, ${ }^{38}$ although more precise measures of sympathetic nervous activity should be used in order to clarify this issue. Thus, a potential contributing mechanism through which intermittent walking breaks attenuated increased fatigue may relate to the maintenance of a balanced regulation of the autonomic nervous system activity. In addition, walking breaks may also counteract fatigue by direct effects on the central nervous system, in which the increased heart rate may serve to increase the delivery of glucose to active domains in the brain. ${ }^{39}$ Functional neuroimaging studies have suggested that the basal ganglia (midbrain) and regions interacting with the basal ganglia play key roles in fatigue both in fatigued and healthy individuals. ${ }^{40}$ The use of these techniques in future studies has the potential to provide significant advances in understanding whether similar brain mechanisms may also mediate the effect of intermittent walking breaks observed in this study.

In contrast to previous research that has used higher break frequencies, ${ }^{1}$ the 3 min walking breaks performed twice an hour in this study did not induce a significant reduction in postprandial glucose levels, indicating that a greater/more regular stimulus may be required to reduce postprandial glucose in the studied population. However, we cannot exclude that the difference in glucose response compared to the previous trial may to some extent relate to differences between interstitial glucose and plasma glucose (which was measured in the previous trial). Comparisons between the two methods have revealed a time lag in interstitial glucose of $10-20$ min. ${ }^{41}$ This time lag may increase when there is a rapid rise in the plasma glucose $^{41}$ and decrease during physical activity due to activity-induced augmented blood flow ${ }^{42}$ and may explain why a more rapid increase in postprandial interstitial glucose was observed in the active condition compared to the sedentary condition in the current trial.

In many countries, increasing time is spent seden$\operatorname{tary}^{43}$ and there is a high prevalence of persistent fatigue. ${ }^{44}$ We examined the effects of light-intensity walking breaks on acute fatigue and whether these results may have implications for persistent fatigue is uncertain. In the prolonged uninterrupted sitting condition, the natural behavioural response to acute fatiguerest-did not seem to reduce the fatigue. Consequently, it could be speculated that uninterrupted sitting may potentially lead to a vicious cycle of fatigue and further sedentary behaviour. Indeed, emerging research has found an association between time spent sedentary and poor sleep efficiency, ${ }^{45}$ which in turn may lead to increased fatigue during waking hours. ${ }^{46}$ Intermittent 


\begin{tabular}{|c|c|c|c|c|c|c|c|c|}
\hline \multirow[b]{2}{*}{ Marker } & \multirow[b]{2}{*}{ Condition } & \multicolumn{6}{|l|}{ Time (h) } & \multirow[b]{2}{*}{ p Value* } \\
\hline & & 0 & $\mathbf{N}$ & 4 & $\mathbf{N}$ & 7 & $\mathbf{N}$ & \\
\hline \multirow[t]{3}{*}{ Heart rate (bpm) } & Active & $64.7(8.1)$ & 19 & $71.8(10.0)$ & 19 & $71.6(11.2)$ & 19 & \\
\hline & Sedentary & $63.5(8.6)$ & 19 & $66.2(9.4)$ & 19 & $66.1(9.7)$ & 19 & \\
\hline & & & & & & & & 0.038 \\
\hline Systolic blood & Active & 119.5 (10.3) & 19 & 117.5 (11.9) & 19 & 117.5 (11.1) & 19 & \\
\hline \multirow[t]{2}{*}{ pressure $(\mathrm{mm} \mathrm{Hg})$} & Sedentary & 119.9 (12.7) & 19 & 117.5 (12.7) & 19 & 119.7 (12.9) & 19 & \\
\hline & & & & & & & & 0.801 \\
\hline Diastolic blood & Active & 76.1 (7.9) & 19 & $74.2(9.3)$ & 19 & $75.3(7.2)$ & 19 & \\
\hline \multirow{2}{*}{ pressure $(\mathrm{mm} \mathrm{Hg})$} & Sedentary & 77.9 (8.6) & 19 & $72.1(9.6)$ & 19 & $74.8(8.8)$ & 19 & \\
\hline & & & & & & & & 0.223 \\
\hline \multirow[t]{3}{*}{ Insulin ( $\mu \mathrm{U} / \mathrm{mL})$} & Active & $21.7(9.2)$ & 18 & 105 (48.54) & 19 & $17.8(6.57)$ & 18 & \\
\hline & Sedentary & $20.9(7.84)$ & 19 & $96.4(51.76)$ & 19 & $19.2(6.52)$ & 19 & \\
\hline & & & & & & & & 0.679 \\
\hline \multirow[t]{3}{*}{ IL-6 (pg/mL) } & Active & $0.95(0.93)$ & 18 & $1.32(0.85)$ & 19 & $1.92(1.42)$ & 18 & \\
\hline & Sedentary & $0.94(0.7)$ & 19 & $1.21(0.51)$ & 19 & $1.34(0.79)$ & 19 & \\
\hline & & & & & & & & 0.123 \\
\hline \multirow{3}{*}{ Cortisol (pg/mL) } & Active & 3452 (3038) & 18 & 1512 (1226) & 19 & 1364 (737) & 18 & \\
\hline & Sedentary & 3618 (5472) & 19 & $1651(1085)$ & 19 & 2653 (3617) & 19 & \\
\hline & & & & & & & & 0.611 \\
\hline \multirow[t]{3}{*}{ BDNF (pg/mL) } & Active & $14905(8324)$ & 15 & 15201 (8628) & 16 & 12605 (7603) & 14 & \\
\hline & Sedentary & 14226 (6852) & 16 & $13098(6854)$ & 15 & $14180(7172)$ & 15 & \\
\hline & & & & & & & & 0.184 \\
\hline \multicolumn{9}{|l|}{ Catechols } \\
\hline \multirow[t]{3}{*}{ Norepinephrine (pg/mL) } & Active & 427 (220) & 18 & $475(238)$ & 18 & 440 (195) & 19 & \\
\hline & Sedentary & 375 (192) & 18 & $466(281)$ & 17 & $473(250)$ & 18 & \\
\hline & & & & & & & & 0.177 \\
\hline \multirow[t]{3}{*}{ DHPG (pg/mL) } & Active & 1244 (292) & 18 & 1259 (307) & 18 & $1276(298)$ & 19 & \\
\hline & Sedentary & $1243(245)$ & 18 & $1338(381)$ & 17 & 1349 (329) & 18 & \\
\hline & & & & & & & & 0.447 \\
\hline \multirow[t]{3}{*}{ Epinephrine (pg/mL) } & Active & $36.6(48.35)$ & 17 & $109.1(226.7)$ & 17 & $171.2(274.89)$ & 18 & \\
\hline & Sedentary & $44.4(54.31)$ & 18 & 118.7 (355.09) & 17 & $69.8(99.55)$ & 18 & \\
\hline & & & & & & & & 0.198 \\
\hline \multirow[t]{3}{*}{ DOPA (pg/mL) } & Active & 1884 (497) & 18 & $1616(455)$ & 18 & 1862 (557) & 19 & \\
\hline & Sedentary & 1919 (505) & 18 & $1739(476)$ & 17 & $2051(588)$ & 18 & \\
\hline & & & & & & & & 0.676 \\
\hline \multirow[t]{3}{*}{ Dopamine (pg/mL) } & Active & 23.7 (26.31) & 18 & $63(158.12)$ & 18 & $42.4(75.24)$ & 19 & \\
\hline & Sedentary & $49.6(134.01)$ & 18 & $39.6(81.37)$ & 17 & $61.2(190.59)$ & 18 & \\
\hline & & & & & & & & 0.234 \\
\hline \multirow{2}{*}{ DHPG/norepinephrine } & Active & $4.0(1.78)$ & 18 & $3.4(1.28)$ & 17 & $3.3(1.34)$ & 18 & \\
\hline & Sedentary & $3.4(1.46)$ & 18 & $3.0(1.01)$ & 18 & $3.2(1.15)$ & 19 & \\
\hline
\end{tabular}


light-intensity walking breaks may be a feasible way to reduce fatigue, especially for individuals with low uptake of MVPA, but the role for walking breaks in the prevention of persistent fatigue needs to be examined in longterm interventions.

This is the first experimental study to examine the impact of walking breaks on cognition under conditions that might correspond to a typical sedentary office or domestic day. The effect on fatigue was not translated into a statistically significant effect on cognitive performance, contrary to what could be expected from previous research on fatigue and cognition. ${ }^{47}$ There are several possible explanations for the lack of effect on cognition. First, the cognitive effect from repeated 3 min bouts of light-intensity physical activity may be too weak to be detected with our sample size. The optimal intensity of the physical activity for cognitive effects is still under debate. For example, the inverted U-hypothesis suggests that moderate intensity physical activity yields the largest effect while 'drive' theories predict that vigorous intensity will be most beneficial. ${ }^{21} 48$ A meta-analysis indicated that when performed directly after the physical activity, lighter (very light, light and moderate) activity is more beneficial, but vigorous intensity is necessary for effects to be maximised if there is a delay between the physical activity and the cognitive test. ${ }^{21}$ Second, it is most likely that reduced fatigue over time may also lead to improved cognitive functioning; however, this needs to be evaluated in longitudinal studies. Third, the cognitive score improved over the day in both conditions, indicating a practice effect, a common problem in cognitive studies with repeated assessment which may have distorted the validity of performance outcomes. Potentially, the cognitive effect from walking breaks may also be moderated by age, fitness level, educational level and the activity performed between tests (doing strict office work or leisure activities such as reading, watching television).

There are several limitations to the study. First, given that this is a pilot study, it was most likely underpowered to detect an effect in cognitive performance (as well as several of the potential mediators). Second, we studied acute effects, and thus any long-term effects of walking breaks cannot be evaluated on the basis of the results in the study. Third, our objective assessments indicated that the participants on average were engaged in MVPA $>30 \mathrm{~min} /$ day, which may have dampened the effect of prolonged sitting during the trial. Finally, we did not investigate whether the effects on fatigue and cognition may be moderated by participant characteristics. We suggest that future studies should: (1) include a larger sample size, (2) conduct repeated cognitive training sessions before the experiment to minimise the practice effect, (3) include a higher frequency of regular blood collection time points to enable more robust analyses of changes over time and, (4) apply functional neuroimaging techniques to increase the knowledge on neurophysiological mechanisms for the relationships between prolonged sitting, light-walking breaks, fatigue and cognition.

In conclusion, intermittent light-walking breaks resulted in an attenuation of fatigue levels during uninterrupted sitting; however, the difference in fatigue did not translate into significantly improved cognitive performance. Our findings provide further support to the suggestion that the relationship between sedentary behaviour and fatigue may be causal and that light-intensity walking breaks may counteract increased fatigue. Although this study only examined short-term effects, there may be longer term relevance and implications for sedentary behaviour, particularly among office workers and others with highly sedentary occupations. Thus, the role that reduced sedentary behaviour and walking breaks may play in the prevention and/or treatment of fatigue warrants further investigation in a full-scale study. Considering the current high prevalence of persistent fatigue in the general population, reductions in sedentary time may have considerable relevance from a public health perspective.

Author affiliations

${ }^{1}$ Department of Public Health and Clinical Medicine, Umeå University, Umeå, Sweden

${ }^{2}$ CEDAR, Center for Demographic and Aging Research, Umeå University, Umeå, Sweden

${ }^{3}$ Baker IDI Heart and Diabetes Institute, Melbourne, Australia

${ }^{4}$ School of Sport Science, Exercise \& Health, University of Western Australia, Perth, Australia

${ }^{5}$ Central Clinical School, Monash University, Melbourne, Australia

${ }^{6}$ The Academic Unit for Psychiatry of Old Age, Department of Psychiatry, University of Melbourne, Melbourne, Australia

${ }^{7}$ School of Population Health, The University of Queensland, Brisbane, Australia

${ }^{8}$ Melbourne School of Population and Global Health, The University of Melbourne, Melbourne, Australia

${ }^{9}$ School of Exercise \& Nutrition Sciences, Deakin University, Melbourne, Australia

${ }^{10}$ Mary MacKillop Institute for Health Research, Australian Catholic University, Melbourne, Australia

${ }^{11}$ Department of Epidemiology and Preventive Medicine, Monash University, Melbourne, Australia

Contributors PW, DWD, C-JB, RL and GL contributed to the conception and design of the study. PW coordinated the trial and data collection, analysed the data, wrote the paper draft and is the study guarantor. MW assisted in collecting the data. PS and JO wrote the statistical analysis plan and conducted the statistical analyses. C-JB, MW, BH, PCD, GL, NE, RL, PS, J0, $\mathrm{JH}-\mathrm{B}, \mathrm{KAE}, \mathrm{NO}$ and DWD analysed the data and reviewed and edited the paper draft. All authors approved the final version of the manuscript.

Funding The study was supported by grants from the Swedish Research Council for Health, Working Life and Welfare, the County Council of Västerbotten, the Foundation for Medical Research in Skellefteå, National Health and Medical Research Council (NHMRC number 569940) and an NHMRC Centre of Research Excellence Grant (NHMRC number 1041056). This work was also partially supported by an OIS grant from the Victorian State Government. DWD is supported by a National Health and Medical Research Council Senior Research Fellowship (number 1078360). NO is supported by an NHMRC Senior Principal Research Fellowship (NHMRC number 1003960). BH is supported by a National Health and Medical Research Counci//National Heart Foundation postgraduate scholarship (number 1056320). GL is supported by an NHMRC Senior Research Fellowship (NHMRC number 1042492). The laboratory of GL currently or recently received research funding from Medtronic, Abbott Pharmaceuticals, Servier Australia and Allergan. GL has acted as a consultant for Medtronic 
and has received honoraria or travel support for presentations from Pfizer, Wyeth Pharmaceuticals, Servier and Medtronic.

Competing interests None declared.

Patient consent Obtained.

Ethics approval The Alfred Hospital Ethics Committee.

Provenance and peer review Not commissioned; externally peer reviewed.

Data sharing statement No additional data are available.

Open Access This is an Open Access article distributed in accordance with the Creative Commons Attribution Non Commercial (CC BY-NC 4.0) license, which permits others to distribute, remix, adapt, build upon this work noncommercially, and license their derivative works on different terms, provided the original work is properly cited and the use is non-commercial. See: http:// creativecommons.org/licenses/by-nc/4.0/

\section{REFERENCES}

1. Dunstan DW, Kingwell BA, Larsen R, et al. Breaking up prolonged sitting reduces postprandial glucose and insulin responses. Diabetes Care 2012;35:976-83.

2. Bailey DP, Locke CD. Breaking up prolonged sitting with light-intensity walking improves postprandial glycemia, but breaking up sitting with standing does not. J Sci Med Sport 2015;18:294-8.

3. Larsen RN, Kingwell BA, Sethi $P$, et al. Breaking up prolonged sitting reduces resting blood pressure in overweight/obese adults. Nutr Metab Cardiovasc Dis 2014;24:976-82.

4. Howard BJ, Fraser SF, Sethi P, et al. Impact on hemostatic parameters of interrupting sitting with intermittent activity. Med Sci Sports Exerc 2013:45:1285-91.

5. Riby LM, McLaughlin J, Riby DM, et al. Lifestyle, glucose regulation and the cognitive effects of glucose load in middle-aged adults. $\mathrm{Br} \mathrm{J}$ Nutr 2008:100:1128-34.

6. Nilsson A, Radeborg K, Björck I. Effects of differences in postprandial glycaemia on cognitive functions in healthy middle-aged subjects. Eur J Clin Nutr 2009;63:113-20.

7. Davenport MH, Hogan DB, Eskes GA, et al. Cerebrovascular reserve: the link between fitness and cognitive function? Exerc Sport Sci Rev 2012;40:153-8.

8. Zoladz JA, Pilc A. The effect of physical activity on the brain derived neurotrophic factor: from animal to human studies. J Physiol Pharmacol 2010;61:533-41.

9. Erickson KI, Voss MW, Prakash RS, et al. Exercise training increases size of hippocampus and improves memory. Proc Nat/ Acad Sci U S A 2011;108:3017-22.

10. Miyashita T, Williams CL. Epinephrine administration increases neural impulses propagated along the vagus nerve: role of peripheral beta-adrenergic receptors. Neurobiol Learn Mem 2006;85:116-24.

11. Carson V, Kuzik N, Hunter S, et al. Systematic review of sedentary behavior and cognitive development in early childhood. Prev Med 2015;78:115-22.

12. Hamer M, Stamatakis E. Prospective study of sedentary behavior, risk of depression, and cognitive impairment. Med Sci Sports Exerc 2014;46:718-23.

13. Parry $S$, Straker $L$. The contribution of office work to sedentary behaviour associated risk. BMC Public Health 2013;13:296.

14. Thorp AA, Kingwell BA, Owen N, et al. Breaking up workplace sitting time with intermittent standing bouts improves fatigue and musculoskeletal discomfort in overweight/obese office workers. Occup Environ Med 2014;71:765-71.

15. Dutta N, Koepp GA, Stovitz SD, et al. Using sit-stand workstations to decrease sedentary time in office workers: a randomized crossover trial. Int J Environ Res Public Health 2014;11:6653-65.

16. Schofield WN. Predicting basal metabolic rate, new standards and review of previous work. Hum Nutr Clin Nutr 1985;39(Suppl 1):5-41.

17. Winkler EA, Gardiner PA, Clark BK, et al. Identifying sedentary time using automated estimates of accelerometer wear time. Br J Sports Med 2012;46:436-42.

18. Healy GN, Dunstan DW, Salmon J, et al. Objectively measured light-intensity physical activity is independently associated with 2-h plasma glucose. Diabetes Care 2007;30:1384-9.

19. Freedson PS, Melanson E, Sirard J. Calibration of the Computer Science and Applications, Inc. accelerometer. Med Sci Sports Exerc 1998;30:777-81.

20. Lee KA, Hicks G, Nino-Murcia G. Validity and reliability of a scale to assess fatigue. Psychiatry Res 1991;36:291-8.
21. Chang YK, Labban JD, Gapin Jl, et al. The effects of acute exercise on cognitive performance: a meta-analysis. Brain Res 2012;1453:87-101.

22. McMorris T, Hale BJ. Differential effects of differing intensities of acute exercise on speed and accuracy of cognition: a meta-analytical investigation. Brain Cogn 2012;80:338-51.

23. Persson J, Kalpouzos G, Nilsson LG, et al. Preserved hippocampus activation in normal aging as revealed by fMRI. Hippocampus 2011;21:753-66.

24. Eriksen BA, Eriksen CW. Effects of noise letters upon identification of a target letter in a nonsearch task. Percept Psychophys 1974;16:143-9.

25. Stroop JR. Studies of interference in serial verbal rections. J Exp Psychol 1935;18:643-62.

26. Kirchner WK. Age differences in short-term retention of rapidly changing information. J Exp Psychol 1958;55:352-8.

27. Dahlin E, Nyberg L, Bäckman L, et al. Plasticity of executive functioning in young and older adults: immediate training gains, transfer, and long-term maintenance. Psychol Aging 2008;23:720-30.

28. Beck RW, Calhoun P, Kollman C. Use of continuous glucose monitoring as an outcome measure in clinical trials. Diabetes Technol Ther 2012;14:877-82.

29. Terada T, Loehr S, Guigard E, et al. Test-retest reliability of a continuous glucose monitoring system in individuals with type 2 diabetes. Diabetes Technol Ther 2014;16:491-8.

30. Lambert GW, Jonsdottir IH. Influence of voluntary exercise on hypothalamic norepinephrine. J Appl Physiol (1985) 1998;85: 962-6.

31. Borg GA. Psychophysical bases of perceived exertion. Med Sci Sports Exerc 1982:14:377-81.

32. Ellingson LD, Kuffel AE, Vack $\mathrm{NJ}$, et al. Active and sedentary behaviors influence feelings of energy and fatigue in women. Med Sci Sports Exerc 2014;46:192-200.

33. Faber LG, Maurits NM, Lorist MM. Mental fatigue affects visual selective attention. PLOS ONE 2012;7:e48073.

34. Demyttenaere K, De Fruyt J, Stahl SM. The many faces of fatigue in major depressive disorder. Int J Neuropsychopharmacol 2005;8:93-105.

35. Klimas NG, Broderick G, Fletcher MA. Biomarkers for chronic fatigue. Brain Behav Immun 2012;26:1202-10.

36. Van Cauwenbergh D, Nijs J, Kos D, et al. Malfunctioning of the autonomic nervous system in patients with chronic fatigue syndrome: a systematic literature review. Eur J Clin Invest 2014:44:516-26.

37. Tanaka M, Mizuno K, Yamaguti K, et al. Autonomic nervous alterations associated with daily level of fatigue. Behav Brain Funct 2011:7:46.

38. Izzo JL Jr, Thompson DA, Horwitz D. Plasma dihydroxyphenylglycol (DHPG) in the in vivo assessment of human neuronal norepinephrine metabolism. Life Sci 1985;37:1033-8.

39. Kennedy DO, Scholey AB. Glucose administration, heart rate and cognitive performance: effects of increasing mental effort. Psychopharmacology (Berl) 2000;149:63-71.

40. DeLuca J, Genova HM, Capili EJ, et al. Functional neuroimaging of fatigue. Phys Med Rehabil Clin N Am 2009;20:325-37.

41. Cengiz E, Tamborlane WV. A tale of two compartments: interstitial versus blood glucose monitoring. Diabetes Technol Ther 2009;11 (Suppl 1):S11-16.

42. Yardley JE, Sigal RJ, Kenny GP, et al. Point accuracy of interstitial continuous glucose monitoring during exercise in type 1 diabetes. Diabetes Technol Ther 2013;15:46-9.

43. Ng SW, Popkin BM. Time use and physical activity: a shift away from movement across the globe. Obes Rev 2012;13:659-80.

44. Junghaenel DU, Christodoulou C, Lai JS, et al. Demographic correlates of fatigue in the US general population: results from the patient-reported outcomes measurement information system (PROMIS) initiative. J Psychosom Res 2011;71:117-23.

45. Pepin V, Boucetta S, Chan-Thim E, et al. The role of sleep and physical activity on the risk for cardiovascular disease. Curr Cardiovasc Risk Rep 2014;413.

46. Chasens ER, Umlauf MG, Weaver TE. Sleepiness, physical activity, and functional outcomes in veterans with type 2 diabetes. Appl Nurs Res 2009;22:176-82.

47. Boksem MA, Meijman TF, Lorist MM. Effects of mental fatigue on attention: an ERP study. Brain Res Cogn Brain Res 2005;25:107-16.

48. Murray NP, Russoniello C. Acute physical activity on cognitive function: a heart rate variability examination. Appl Psychophysiol Biofeedback 2012;37:219-27. 